BONPLANDIA 20(2). 2011

ISSN: 0524-0476

\title{
PASADO Y PRESENTE DEL USO DE PLANTAS SILVESTRES CON ÓRGANOS DE ALMACENAMIENTO SUBTERRÁNEOS COMESTIBLES EN LA PATAGONIA
}

\author{
JUAN J. OCHOA ${ }^{1} \&$ ANA H. LADIO²
}

Summary: Ochoa, J. J. \& A. Ladio 2011.Past and present use of wild plants with edible underground storage organs in Patagonia. Bonplandia 20(2): 265-284.

\begin{abstract}
The present work is a first approach to the study of the role of edible plants with underground storage organs (PUSO) in Patagonia, from both temporal and spatial perspectives, integrating ethnohistorical and current ethnographic data on the use of PUSO. Of the 54 sources studied, $75 \%$ mention the use of wild PUSO for human alimentation in Patagonia. There are 51 species of wild edible PUSO registered, the most common group being those with edible roots. The steppe and transition zones present the greatest richness of PUSO, probably because of ecological adaptations associated with drought in the former case, and the relatively high diversity typical of ecotones in the latter. Marked discontinuity of use was revealed by ethnohistorical registers, with few species present for longer than a century. Worthy of special mention are the documented continuity of $A$. tuberosa from the18th century up to the present time, and the recent incorporation of exotic species. When the ethnohistorical studies reviewed are compared with recent ethnobotanical field studies, dynamic use patterns are suggested, which over time have led to the abandonment of PUSO in alimentary practices in Patagonia. The unusual characteristics found with respect to temporal and spatial factors can be related both to specific local knowledge developed over time by Patagonian inhabitants, and the sociopolitical and academic circumstances of the different literature analysed.
\end{abstract}

Key words: Wild plant resources, temporal and spatial patterns of use, ethnobotany, ethnohistorical data.

Resumen: Ochoa, J. J. \& A. H. Ladio 2011. Pasado y presente del uso de plantas silvestres con órganos subterráneos de almacenamiento comestibles en la Patagonia. Bonplandia 20(2): 265-284.

El presente trabajo es la primara aproximación acerca del papel de las plantas con órganos de almacenamiento subterráneos comestibles (POAS) en la Patagonia, desde una perspectiva temporal y espacial, al integrar datos etnohistóricos y etnográficos actuales ligados al uso de las POAS. El $75 \%$ de las 54 fuentes analizadas mencionan el uso de POAS en la alimentación humana de la Patagonia. Las POAS silvestres comestibles registradas ascienden a 51 especies, siendo mayor el conjunto de POAS con raíces comestibles. La estepa y zonas ecotonales resultaron las de mayor riqueza de POAS, probablemente vinculado a adaptaciones ecológicas de éstas a la aridez en el primer caso, como a la alta diversidad relativa que presentan los ecotonos, en el segundo. El registro etnohistórico mostró discontinuidades marcadas, siendo pocas las especies presentes por más de un siglo, destacándose la continuidad documental

${ }^{1}$ Instituto de Investigaciones en Diversidad Cultural y Procesos de Cambio (IIDyPCa) Universidad Nacional de Río Negro. Mitre 630, 5to Piso. Bariloche (8400). Río Negro, Argentina. juanochoa10@gmail.com

${ }^{2}$ Instituto de Investigaciones en Biodiversidad y Medio Ambiente (INIBIOMA). Universidad Nacional del Comahue. Quintral 1250. Bariloche (8400). Río Negro, Argentina. aladio2002@yahoo.com.ar 
de A. tuberosa desde el siglo XVIII al presente, así como la incorporación reciente de especies exóticas. Las fuentes etnohistóricas revisadas y su contraste con datos etnobotánicos recientes de campo sugieren dinamismo en los patrones de utilización, que a lo largo del tiempo han llevado al abandono del uso de las POAS en la práctica alimentaria de la Patagonia. Las singularidades encontradas respecto a lo temporal y espacial pueden vincularse tanto a los conocimientos locales y específicos desarrollados a lo largo del tiempo por los habitantes de la Patagonia, como también a las circunstancias sociopolíticas y académicas de cada una de las fuentes analizadas.

Palabras claves: Recursos vegetales silvestres, uso temporal y espacial, etnobotánica, datos etnohistóricos.

\section{Introducción}

El interés por estudiar a las plantas con órganos de almacenamiento subterráneos (POAS) y su relación con poblaciones "humanas" de la Patagonia surge a raíz de las siguientes observaciones: a) la recolección de este tipo de recurso ha sido señalada como una constante para diferentes grupos humanos alrededor de todo el mundo (Hladik \& al., 1984; Odea, 1991; Peters \& Obrien, 1981); b) dado que estos órganos subterráneos acumulan agua, almidones, proteínas y vitaminas, compuestos esenciales para las dietas humanas, y pueden resultar de gran importancia en regiones donde escasea la oferta de carbohidratos vegetales, como en la Patagonia árida; c) en esta región el uso de POAS fue documentado tempranamente por los primeros exploradores europeos a partir del siglo XVI, y aparecen en los relatos y crónicas de diferentes exploradores y aventureros durante los siglos siguientes (Vignati, 1941); d) los trabajos etnográficos realizados a partir de mediados del siglo XX hasta el presente, dan cuenta de la vigencia de conocimientos acerca de dichos recursos, señalando además que las prácticas de recolección están siendo abandonadas (Ladio, 2006; Martínez Crovetto, 1980); y finalmente, e) existen pocos estudios de carácter etnobotánico y ecológico sobre las POAS en la Patagonia.

Partimos de la idea de que, como cualquier otro recurso natural presente en una región habitada por el ser humano, los conocimientos y prácticas asociadas al uso de POAS son dinámicos y dependen del contexto ecológico cultural (Berkes \& Folke, 2002; Ladio, 2011a). Así, a lo largo del tiempo de ocupación de la Patagonia estos recursos han de haber tenido un papel diferencial tanto si consideramos a las sociedades de cazadores recolectores, como a las sociedades que surgieron tras el contacto cultural con los colonizadores europeos o a las sociedades campesinas actuales. Cada sociedad se caracteriza por pautas culturales propias (ej. tabúes, reglas consuetudinarias, etc.) (Cruz Cruz, 1991; Ladio, 2011b) que determinan cuáles plantas son comestibles, de qué manera y cuándo se comen. Estas pautas alimentarias, en los grupos indígenas de la Patagonia, han cambiado a lo largo del tiempo a raíz de la introducción de nuevos recursos, la imposición de prácticas alimentarias tras la conquista, y la hibridación de saberes tradicionales con nuevos conocimientos provenientes de los inmigrantes europeos, o de las sociedades que emergieron tras la formación de los estados argentino y chileno (Eyssartier \& al., 2011; Torrejón \& Cisternas, 2002).

Junto a estas variaciones asociadas al momento histórico cultural, existen diferencias en relación al contexto ecológico vinculado a las distintas heterogeneidades espaciales y biogeográficas que ofrecen distintos sets de recursos. Por ejemplo, se ha señalado que las plantas silvestres son incluidas en la dieta en función de múltiples factores como su abundancia o apariencia local (Albuquerque \& Lucena, 2005; Ochoa \& al., 2010a), facilidad de encuentro (Phillips \& Grentry, 1993), calidad nutricional y retorno energético (Ladio \& Lozada, 2000, 2004), estacionalidad (Marlowe \& Berbesque, 2009; Peters \& al., 1984).

En este trabajo, desde una perspectiva proveniente principalmente de la Etnobotánica histórica, es decir, a partir de la mirada de trabajos realizados en el pasado por otros autores junto con el aporte de trabajo 
etnobotánico en comunidades actuales, se propone responder las siguientes preguntas:

¿Cuál es, en el registro bibliográfico, la riqueza acumulada de POAS utilizadas como alimento por las distintas culturas en Patagonia? y ¿qué características poseen?

¿Qué papel jugaron estos recursos según la visión de los autores en el pasado?

¿Cómo varía su distribución en el gradiente fitogeográfico?

¿Cómo ha variado la riqueza de especies citadas en el gradiente temporal?

¿Qué continuidades y discontinuidades a nivel de especies se evidencian a través del registro bibliográfico y etnográfico?

Este trabajo pretende, por un lado, caracterizar el registro bibliográfico sobre el uso de las POAS en la región, y, por el otro, demostrar la importancia de las aproximaciones etnohistóricas en los estudios etnobotánicos como herramientas claves para comprender de manera integrada el uso de los recursos naturales en el pasado y el presente.

\section{Materiales y Métodos}

\section{Revisión de fuentes históricas}

Existe una discontinuidad (en lo temporal y espacial) del registro escrito sobre los indígenas que habitaban la Patagonia entre los siglos post-colonización y el presente. En este trabajo, las fuentes se ordenaron de manera arbitraria en períodos seculares. Las POAS utilizadas por los diferentes grupos indígenas patagónicos post-conquista y las prácticas alimentarias asociadas se investigaron a través de la revisión de un total de 54 fuentes que corresponden a: 9 del siglo XVI, 7 del siglo XVII, 8 del siglo XVIII; 12 del siglo XIX, 11 del siglo XX y 7 del siglo XXI.

Cada una de las fuentes mencionadas fue revisada en detalle, consignando todo dato referido a costumbres alimentarias que incluyeran referencias al uso de POAS. Las fuentes bibliográficas fueron analizadas sin considerar las particularidades y complejidades culturales de cada una de las sociedades que habitaron la Patagonia, dado que el principal objetivo fue detectar el carácter utilitario de las POAS y su variación en el espacio y el tiempo. Para cada referencia se confeccionó una tabla que incluye: nombre asignado en la fuente, nombre científico, la región donde fue observado su uso, y el tipo de órgano subterráneo presente (Medeiros, 2009). A continuación se describe con mayor detalle las principales características de los trabajos analizados:

Siglo XVI. Se trata del siglo inicial de contacto entre los indígenas patagónicos y el hombre europeo. Es el período de menos fuentes disponibles, no por eso menos interesantes, ya que en éstas se reflejan costumbres sin las transformaciones culturales que el contacto ocasionaría posteriormente. Desde la excursión de Magallanes, iniciada en el año 1512, y la crónica dejada por Pigafetta en 1536 (Pigafetta, 1941), consideramos la de Juan de Mori (1889) y Alonso Vehedor (1866) que integraron la expedición de Simón de Alcazaba que, en 1535, desembarcó en la actual Bahía de Camarones y fundó la Gobernación de Nueva León; la de Fernández Oviedo y Valdés que escribió sobre la Patagonia en 1535 (1944); la de Tomé Hernández (1950), sobreviviente de la expedición de Sarmiento de Gamboa en 1579. Por último, se revisó la crónica de Jerónimo de Vivar, escrita en 1524 (1966), la de Góngora de Marmolejo de 1576 (1990) y la de Mariño de Lobera de 1580 (1865), todos soldados cronistas de Pedro de Valdivia, que dejaron observaciones sobre la vertiente occidental de los Andes.

Siglo XVII. Para este período los documentos se originaron principalmente en el noroeste patagónico, dado el impulso misionero de la época, así como las motivaciones por encontrar la legendaria ciudad de los Césares. Se revisaron: las relaciones de Gerónimo Luis de Cabrera que entre 1620-1621 recorrió la zona del Nahuel Huapi (Nocceti \& Mir, 2000), contemporáneas de la dejada por el capitán Juan Fernández, descubridor del lago Nahuel Huapí (Schobinger, 1958-1959), cuyo derrotero nos llegó a través del Memorial de Don Diego Flores de León (reproducido en Vignati, 1939). También las obras de los siguientes misioneros jesuitas: Diego de Rosales, quien visitó la zona del Nahuel Huapi en 1653 (1877); la carta escrita en 1670 
por el Padre Nicolás Mascardi, fundador de la Misión de Nahuel Huapi reproducida por Vignati (1963). Además, se revisaron las obras de Alonso Ovalle (1646), Alonso González de Nájera (1889) y Francisco Núñez de Pineda y Bascuñán (1861), quienes escribieron sobre las poblaciones indígenas en la vertiente occidental de los Andes patagónicos.

Siglo XVIII. Para este siglo se trabajó con las fuentes dejadas en 1703 por el padre Felipe Vander Meer o Felipe de Laguna (1930), quien se hizo cargo de la misión del Nahuel Huapi entre 1703 y 1707; la "Historia de la Compañía de Jesús en Chile", escrita por Miguel de Olivares (1878) entre 1736 y 1738, donde refiere su viaje a Nahuel Huapi entre los años 1706 y 1707. Se cuenta con el testimonio de Bernardo Havestadt, misionero jesuita procedente de Chile, quien realizó un viaje entre 1751 y 1752 con el objeto de tomar contacto con los pehuenches de las pampas, al otro lado de la cordillera (Havestadt, [17511752] 1930). También se revisó el viaje del jesuita Thomas Falkner (1911); el relato de Basilio Villarino (1972) en su viaje por el Río Negro y el de Francisco de Viedma (1783) por la costa atlántica. Se revisaron las crónicas de los viajes efectuados, entre 1791 y 1794, por Francisco Menéndez, misionero franciscano proveniente de Chile y encargado de reconocer la zona aledaña al Nahuel Huapi después de la expulsión de los jesuitas (Menéndez, [17911794] 1896); el diario de Francisco González referido al viaje que hiciera por tierra entre Puerto Deseado y el Río Negro en 1794. Por último, se consultó las obras de Antonio Machoni (1731), José Sánchez Labrador (1936) y la de Juan Ignacio Molina (1878), las cuales hacen referencia a este siglo.

Siglo XIX. Para la primera mitad del siglo XIX se analizó el itinerario seguido por Luis de la Cruz en 1806, desde Concepción hasta Buenos Aires, quien tomó contacto con los pehuenches y los ranquilinos (Cruz, 1969) y la de Benjamin Bourne (1998) que en 1849 estuvo cautivo por tehuelches meridionales durante noventa días. En cuanto a la segunda mitad del siglo, las fuentes son más abundantes: los viajes de Guillermo Cox en 1862, expedicionario chileno que navegó el Nahuel Huapi y la parte superior del Río
Limay (Cox, 1863); el de Jorge Claraz, explorador suizo que, entre 1865 y 1866 , recorrió la zona norpatagónica llegando hasta Chubut, a través del camino del centro; es decir, la ruta de Valcheta-río Chubut Medio (Claraz, 1988). También, el que llevara a cabo George Musters (1997) entre 1869-1870, desde la Isla Pavón hasta Carmen de Patagones acompañando a una partida de tehuelches meridionales que se dirigían a cobrar las raciones del gobierno argentino y a realizar un importante parlamento; Augusto Guinnard (1947) que entre 1871 y 1873 , estuvo cautivo bajo diferentes parcialidades indígenas; el de Henry de la Vaulx (1901) que en 1896 recorrió la Patagonia desde Carmen de Patagones; el relato de Fitz Roy (1839) y Darwin (1964) referidos a la expedición ocurrida entre los años 1831 y 1836 . Finalmente, los relatos de Francisco P. Moreno en 1875 al "país de las Manzanas" (Moreno, 1969), Ramón Lista (1894) producto de contactos con parcialidades tehuelches, y los de Señoret (1896) y Mayne (1874), quienes exploraron la región magallánica.

Siglos $X X$ y $X X I$. Los trabajos revisados para estos siglos fueron los de carácter etnográfico realizados por Martin Gusinde acerca de las diferentes etnias que habitaban Tierra del Fuego y los canales del Estrecho de Magallanes (Gusinde, 1931), así como de nativos araucanos (Gusinde, 1917); por Carlos Gallardo (1910) sobre los onas; y Anne Chapman (1986) sobre los selkman. Para finalizar la revisión se realizó una sistematización de los trabajos de carácter explícitamente etnobotánico para la región, correspondientes a la segunda mitad del siglo XX hasta el presente: Martínez Crovetto (1968, 1980, 1982); Vignati (1941); Steibel (1997), Gumucio \& Insunza (1992), Villagrán (1998), Ladio (2001, 2006); Ladio \& Lozada (2000, 2004); Ladio \& al. (2007); Ochoa \& al. (2010a,b).

\section{Trabajo de campo}

Se complementó la revisión bibliográfica con información etnobotánica de campo obtenida durante el año 2010. Los sitios de estudio correspondieron a las poblaciones de Lagunita 
Salada, donde viven aproximadamente 100 familias y el Escorial, habitado por alrededor de 30 familias, ambos parajes ubicados en la meseta central de la Provincia de Chubut, correspondiente a la provincia fitogeográfica patagónica (Cabrera, 1976). Además, se trabajó en Villa Llanquín (150 familias), paraje ubicado sobre la costa del Río Limay en la Provincia de Río Negro, y Nahuel Pan (30 familias), en la Provincia de Chubut; ambas zonas corresponden a un ecotono entre la estepa patagónica y los bosques andino-patagónicos (Cabrera, 1976). Todas estas poblaciones se caracterizan por estar conformadas por un alto número de pobladores de ascendencia indígena (mapuche y/o tehuelche), en ambientes rurales donde, principalmente, basan su economía de subsistencia en la cría extensiva de ganado ovino. En cada paraje se entrevistó a 5 habitantes. En total, se realizaron 20 entrevistas con distintos grados de estructuración (libres $\mathrm{y}$ dirigidas), junto con historias de vida $\mathrm{y}$ observación participante (Albuquerque \& al., 2010). Previamente, se estableció con los entrevistados de los diferentes parajes los alcances y las formas de devolución de la información obtenida en esta investigación, así como el consentimiento para trabajar en los parajes (previamente informado). Se entrevistó a una persona de una familia, elegida al azar dentro de cada poblado (Ladio \& Lozada 2000). En este trabajo se documentó sólo parte de la información recabada, con especial énfasis en las especies de POAS citadas por los informantes. Este set de datos fue considerado como una fuente adicional al conjunto de fuentes tratadas del siglo XXI.

\section{Análisis de datos}

Se estableció el número acumulado total de POAS en término de especies, géneros $\mathrm{y}$ familias a lo largo de todas las fuentes analizadas en el gradiente temporal. Por otra parte, se cuantificó la cantidad de fuentes etnohistóricas y la riqueza de especies (POAS) para cada uno de los siglos analizados. La continuidad de las especies fue analizada mediante la suma de recursos que se citan para un siglo, hasta 4 siglos. La riqueza total de POAS encontrada en los relevamientos etnográficos se estableció como la suma de las especies citadas en las cuatro localidades analizadas. Por otra parte, se categorizaron las especies según la pertenencia biogeográfica, considerando el punto geográfico al que hace referencia la fuente bibliográfica y su correspondencia con las diferentes regiones fitogeográficas de Cabrera (1976), recategorizadas por unidad ecológica: Bosque, para la provincia Subantártica; Estepa, para la provincia Patagónica, y Monte para la provincia homónima. Además, cuando las fuentes revisadas hicieron referencia a zonas de transición entre el bosque y la estepa, o la estepa y el monte, fueron re-categorizadas como Ecotono. Cada especie registrada en las fuentes revisadas fue categorizada según a qué tipo de órgano subterráneo correspondía: raíz, bulbo, tubérculo y rizoma (Correa, 19691999). La similitud de plantas registradas entre diferentes siglos se analizó a través del índice de Jaccard (Mueller-Dombois \& Ellenberg, 1974). Este índice se basa en la presencia o ausencia de plantas en la comunidad o conjunto de datos, y manifiesta el número de especies en común con respecto al número total de especies, expresado como JSI $=(\mathrm{c} / \mathrm{a}+$ $\mathrm{b}+\mathrm{c}) \times 100$, donde $\mathrm{c}$ es el número de especies en común, a es el número de las especies que sólo se encuentran en la comunidad A, $\mathrm{y} \mathrm{b}$ el número de especies que solamente se encuentran en la comunidad B. Todos los datos fueron analizados con estadística no paramétrica (Agresti, 1996).

Los registros de audio y notas de campo se encuentran el Laboratorio Ecotono y el material de herbario del trabajo de campo realizado en las poblaciones de Villa Lanquin. Lagunita Salada, Escorial y Nahuel Pan se encuentran depositados en el Herbario BCRU del Centro Regional Universitario Bariloche.

\section{Resultados y Discusión}

\section{Riqueza acumulada de POAS comestibles en la Patagonia}

De las 54 fuentes revisadas, el $75 \%$ mencionan el uso de POAS en la alimentación humana en Patagonia, y el $57 \%$ de las mismas 
registraron nombres locales de etnoespecies. Estos resultados, indicarían que la práctica de recolectar POAS ha sido relevante en la región, apareciendo en los textos en forma recurrente a pesar de que el propósito de la mayoría de los mismos no estuvo vinculado a estudiar esta práctica directamente.

En total, para la región, se documentaron 100 nombres vulgares, de los cuales, 83 han sido identificados científicamente y corresponden a 51 especies, que se distribuyen en 34 géneros y 23 familias botánicas (Tabla 1). Del total de especies, 47 corresponde a especies nativas de la región y 4 son exóticas. La familia con mayor riqueza de POAS es Apiaceae, y el género más representado es Azorella. Otros géneros bien representados, con 3 especies cada uno, son Alstroemeria, Oxalis y Dioscorea (Tabla 1). Diecisiete etnoespecies no han podido ser asignadas a ningún taxón hasta el momento, dado que no existe material de herbario asociado ni descripciones botánicas adecuadas (Tabla 2). Sin embargo, su registro y sistematización es de gran utilidad para futuros estudios etnobotánicos pues brinda información fitonímica, temporal y geográfica sobre recursos comestibles en la región que puede ser contrastada con posteriores trabajos de campo.

Esta lista total de especies, posiblemente incompleta, refleja la importancia de estas POAS como parte de tradiciones alimentarias de distintas culturas patagónicas que exploraron y utilizaron elementos que crecen bajo tierra. Cabe señalar que, el mayor porcentaje corresponde a especies con raíces comestibles (26), seguidas por rizomas (11), tubérculos (10) y bulbos (4) (Fig. 1), tendencia que es similar a la proporción documentada en las Floras de la región (Ochoa \& Ladio, 2010), mostrando en gran medida la estrecha unión entre los recursos disponibles y sus adaptaciones biológicas y los procesos de selección cultural.

El trabajo de campo preliminar realizado

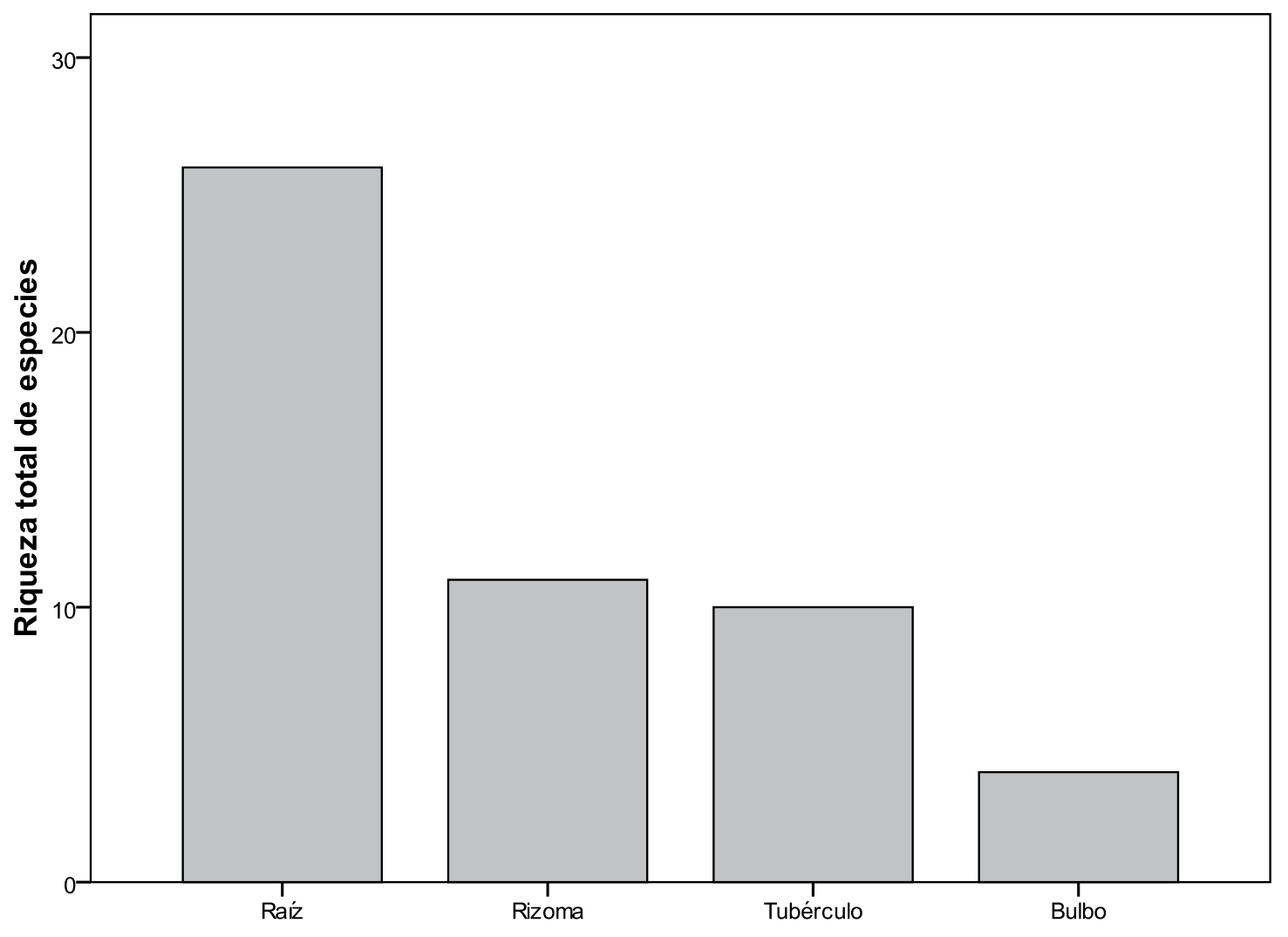

Fig. 1. Tipos de órganos de almacenamiento subterráneos comestibles correspondientes a las 51 especies vegetales registradas en la presente revisión. 
en las poblaciones de Lagunita Salada, El Escorial, Nahuel Pan y Villa Llanquín evidencia un total de 8 especies comestibles de POAS (Tabla 1). Además se citaron dos nombres vulgares que no pudieron ser identificados taxonómicamente (Tabla 2). Resulta interesante que Arjona tuberosa es la única citada en los cuatro parajes, otras, como Azorella monantha y Tropaeolum porifolium fueron citadas solo en las localidades esteparias de Lagunita Salada y El Escorial, mientras que Oxalis adenophylla solo en las poblaciones asentadas en las zonas ecotonales (Nahuel Pan y Villa Lanquín). El resto de las especies sólo fueron citadas en una comunidad.

Este set de datos de campo refuerza la idea de la importancia, no sólo de la disponibilidad de las especies en los patrones de uso de las mismas, sino también del carácter local, circunstancial y específico de los saberes albergados por cada población en el momento del estudio. Por otra parte, los resultados parecen vincularse a la amplitud fitogeográfica de las especies utilizadas. Por un lado, $A$. tuberosa se distribuye en toda la Patagonia, mientras que $O$. adenophylla se encuentra restringida a zonas de alta montaña y de transición entre el bosque y la estepa (Correa, 1969-1999), donde se ubican los parajes en que fueron citadas. Por otro lado, los grupos humanos actuales ya no dependen únicamente de los recursos silvestres, están sujetas a otros patrones alimentarios y necesidades que modelan nuevas formas de uso y apropiación del ambiente.

Asimismo, el trabajo de campo confirma procesos de cambio que acontecen en este cuerpo de conocimientos en donde no sólo se denota pérdida de saberes sino también incorporación de otros nuevos. Por ejemplo, en Villa Llanquín se hizo mención a una especie exótica, Pastinaca sativa, conocida como "pana", la cual aparece citada como comestible en Patagonia exclusivamente para este siglo. La misma fue recolectada en estado silvestre y cultivada en el pasado en la comunidad. Esta especie es originaria de Eurasia, donde sus raíces pivotantes han sido consumidas desde tiempos remotos (Cain \& al., 2010). Si bien no poseemos datos precisos sobre la historia de su introducción en la
Patagonia, su ausencia en el registro histórico puede deberse al reciente ingreso en la región. La incorporación de nuevos recursos puede ser interpretada como una estrategia adaptativa de las poblaciones humanas ante cambios en su ambiente ecológico (extinciones e invasiones biológicas) y transformaciones de su contexto cultural (Torrejón \& Cisternas, 2002).

\section{El papel de las POAS en el registro etnohistórico}

Del análisis de las fuentes históricas se desprenden dos patrones de usos diferenciales. Por un lado, los primeros cronistas mencionaron a este tipo de recurso como base de la alimentación indígena. Por ejemplo, Pigafetta (1941: 70) haciendo referencia a cazadores recolectores que habitaban la estepa patagónica sobre el atlántico relató: ...Su alimento consiste en carne cruda y una raiz que llaman "Capac"... Sobre la vertiente occidental Vivar (1966: 138) dijo para la zona del Río Maule: ...ellos se lo contaron y como sembraban muy poco y se sustentaban el mayor del tiempo de raices de una manera de cebollas que tengo dicho, y de otra raiz que llaman ellos "pique-pique", que es una manera de castañas peladas, salvo que no tienen el gusto que ellas, y blancas.... Las referencias de Molina (1878: 145) también fueron contundentes respecto al uso habitual de "maglias" (Solanum maglia), junto a los cultivos de Solanum tuberosum, así como del "illmu" (Sysirinchium striautum)....Los naturales del país comen la raíz o tubérculo de esta planta en sus sopas y es de un sabor agradable como lo supe de mi propia experiencia... ; González (1965: 140): desde Puerto Deseado hasta el curso del rio Chubut, los indigenas iban a buscar raices para comer...; o Menéndez (1896) en sus referencias al uso de "tucuyu" (Alstroemeria aurea) de forma idéntica al "ligtu" (Alstroemeria ligtu) en la vertiente oriental de los Andes. Las citas que evidenciaron el uso habitual en general se restringen a los primeros siglos de contacto (XVI al XVIII).

El segundo patrón de uso que proponemos, es el de la utilización de estos recursos ante situaciones estacionales o azarosas de escasez 
de otras fuentes alimenticias preferidas. Los ejemplos provienen principalmente de observaciones realizadas a partir del siglo XIX así como los datos etnobotánicos de poblaciones actuales. Rosales (1877: 166) relató que en tiempo de guerra en la Araucanía: ...se acogen a comer yerbas y raices del campo, con que hacen grandes ventajas a los españoles en el sufrimiento de el ambre...; Orbigny (1945: 696): ... En tiempo de carestía, empero, recogen los granos de una planta crucifera, vecina de nuestra "mostaza", que muelen entre dos piedras, antes de comer; o bien hacen asar, en una olla, una raicilla negra y larga, bastante parecida al "diente de perro", que las mujeres apilan después y la convierten en una harina sin sabor, que les sirve provisoriamente. Musters (1997: 88): ... Nuestro charqui habia alcanzado apenas para una comida vespertina; de modo que tuvimos que satisfacer nuestro apetito con la provisión de raices tuberosas de una planta que crece en gran cantidad en casi toda la Patagonia... ; o Moesbach (1930: 22), cuando refiriéndose al "ampe" (Lophosoria quadripinnata) documentó: su rizoma se comía en tiempos de escasez...; Cox (1863: 62): ....Nuestros peones... hacían de tiempo en tiempo cortas paradas al pie de los montecitos de "pangue" (Gunnera tinctoria), y chupaban con mucho gusto el jugo un poco ácido que contienen las raices...

Interesantes resultan las citas de Bourne, quien no sólo hizo referencia al ofrecimiento de raíces por parte de los nativos durante el viaje, sino testimonió cómo estando solo y con hambre se encontró con ejemplares de "bardana"...una maleza humilde pero ¿por qué no probar?...empecé un proceso de "extraer raices" con buen éxito... asé sobre las cenizas calientes... (Bourne, 1998: 92). Las referencias etnohistóricas continúan, pero lo interesante es que se cita como recurso de emergencia.

El trabajo etnográfico actual realizado en las poblaciones de la estepa patagónica y ecotono con el bosque andino, aún cuando se trató de un número pequeño de entrevistados, sugeriría este último patrón de uso de las POAS. Algunas citas de los pobladores mencionaron que tanto en el pasado como en el presente la recolección de papitas o raíces se realiza... sólo cuando se está en el campo con los animales, trabajando con los alambrados, o son los niños los que la recogen cuando juegan... Este patrón de uso coincide parcialmente con trabajos etnobotánicos realizados en otras poblaciones rurales patagónicas (Ladio, 2001, 2002, 2006) que conciben a las "papas silvestres" como alimentos culturalmente importantes del pasado y del presente, pero en proceso de abandono. Asimismo, se señala que su utilización está situada estacionalmente en primavera, cuando la producción de las huertas es casi nula.

Los dos patrones de uso de POAS sugeridos en este trabajo para la Patagonia estarían indicando un progresivo desuso de estos recursos, en general, a lo largo de los siglos analizados, pasando de ser un recurso cotidiano y complementario en la dieta, a un recurso marginal recolectado en situaciones específicas. Este cambio en el patrón puede vincularse a las profundas transformaciones socioculturales que experimentaron los grupos humanos que habitaron la región durante estos siglos. Por un lado, los tempranos cambios hacia una cultura ecuestre (Moreno \& Videla, 2008), la apropiación del ganado y la incorporación de prácticas agrícolas no tradicionales (Torrejón \& Cisternas, 2002) que modificaron las prácticas de caza y recolección, tanto hacia la incorporación de nuevos recursos como al abandono de otros (Ladio, 2011a). Por último, con la consolidación de los estados nacionales, y las diferentes políticas alimentarias oficiales (Fernández \& Crivelli, 2006), se ha favorecido una creciente dependencia hacia productos del mercado, en detrimento del uso de recursos silvestres.

\section{POAS en el gradiente ambiental según el registro etnohistórico}

Cabe señalar que las fuentes etnohistóricas no muestran una indagación exhaustiva de todos los ambientes de la Patagonia por igual, ciertos ambientes y culturas han sido preferidos por los autores por razones geopolíticas y económicas, reflejando el contexto histórico-cultural de cada siglo (Navarro Floria, 2006). Así también el análisis de las fuentes refleja la importancia 
de las vías de acceso para los exploradores (valles, ríos y océanos) que coinciden con las mejores tierras de asentamiento para las sociedades locales patagónicas (Bandieri, 2005). Por ejemplo, se destaca como ejes de interés las exploraciones marítimas en la costa atlántica en el recorrido estratégico hacia el Estrecho de Magallanes (Martinic, 2006), la búsqueda de la ciudad perdida de los Césares o Trapalanga (Bandieri, 2005; Latcham, 1929), la exploración de la zona del Nahuel Huapí, primero con fines evangelizadores, más tarde por el valor económico de sus recursos naturales (Nicoletti, 2004). Asimismo, incluso los trabajos etnobotánicos actuales están orientados hacia zonas esteparias dado que las comunidades originarias, quienes albergan una gran riqueza de saberes sobre los recursos vegetales, han sido desplazadas en mayor medida hacia esos ambientes marginales (Ladio, 2001).

Como consecuencia, las fuentes indagadas aquí muestran una mayor referencia a zonas esteparias y de bosque, mientras que las zonas ecotonales y de monte resultaron con menor representación (Fig. 2). Los datos sugieren que en los ambientes esteparios, caracterizados por su estacionalidad marcada, se ha favorecido la existencia de especies con órganos adaptados al almacenamiento de nutrientes y agua (NoyMeir, 1978) y que han sido aprovechados por sus habitantes como sucede en otras regiones de características similares (Campbell, 1986; Vincent, 1985). Por otra parte, las zonas transicionales entre los territorios provincias fitogeográficas también presentan altos valores de riqueza de especies, lo que podría explicarse por el hecho de que las regiones ecotonales se caracterizan por ser áreas de superposición $\mathrm{y}$, por ende, con mayor diversidad específica (Begon \& al., 2005).

Sin embargo, a pesar del registro fragmentario y la desigual representación de ciertas zonas de la Patagonia en las fuentes, el uso de POAS nos brinda información valiosa respecto a la biogeografía de recursos silvestres comestibles del pasado, que puede resultar clave en el entendimiento de los patrones de movilidad de los grupos humanos en la región.
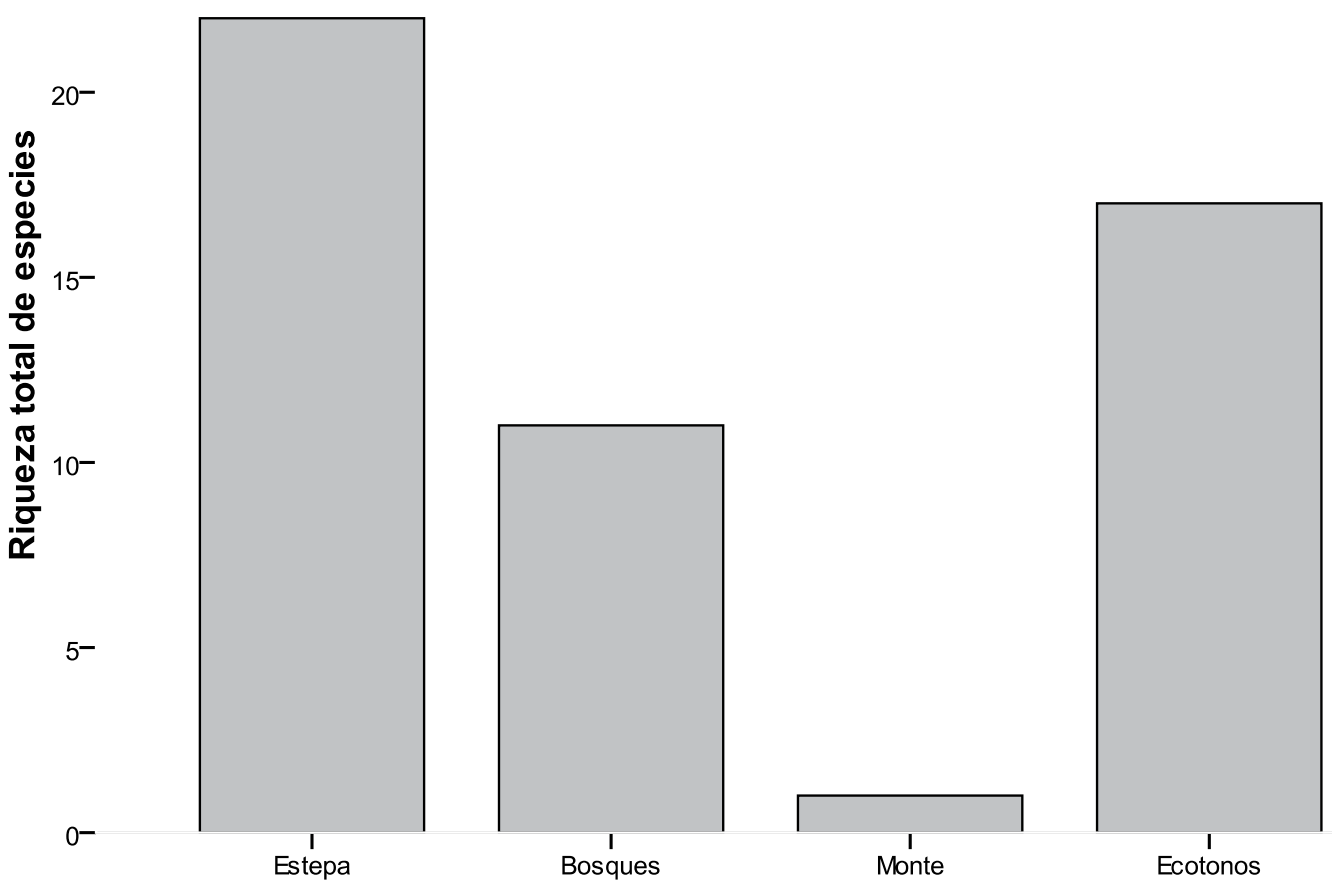

Fig. 2. Riqueza de POAS comestibles categorizadas por ambiente. 


\section{El uso de las POAS en el gradiente temporal: continuidad y discontinuidad en el registro de uso}

Se encontró que la riqueza de especies citadas en los trabajos es significativamente independiente del número de fuentes revisadas para cada siglo (Correlación de Spearman, $\mathrm{p}>0,05$ ), mostrando que ante un número similar de fuentes analizadas para cada período, la riqueza presenta variaciones marcadas que se deben posiblemente a otros factores (Fig. 3). Además, cabe señalar que las fuentes del siglo XVII no documentaron ninguna POAS comestible, por lo que sería necesario indagar las razones de este hiato en los documentos etnohistóricos para ese siglo. Posiblemente, la ausencia de fuentes se deba a que este período fue de alta conflictividad para los grupos locales debido al el incremento de expediciones esclavistas en la región norpatagónica, que impidieron otro tipo de acercamiento (Bandieri, 2005).

Por otra parte, se encontró que la riqueza de especies citada en los trabajos disminuye significativamente con la antigüedad de la fuente (Correlación de Spearman, $r=-0,9$; $\mathrm{p}<0,05)$, con un máximo de riqueza en el siglo XX (Fig. 3). El aumento de la riqueza registrada a lo largo del tiempo se debería posiblemente a dos factores. Por un lado, los registros etnohistóricos desde el siglo XVI hasta fines de siglo XIX no tenían como objetivo principal reconocer los recursos vegetales, ni implicaron metodologías sistemáticas (por ejemplo, entrevistas), pudiendo haber ignorado muchos de los recursos naturales y las prácticas llevadas a cabo por las poblaciones con las que tuvieron contacto. Por otra parte, es de notar que, a partir del siglo XVII, extendiéndose el uso del caballo y del ganado a casi toda la Patagonia, las sociedades locales empezaron a responder a patrones socioeconómicos más complejos que el modelo de caza y recolección antes presente, pero también con mayor asimilación al modelo económico hispano-criollo. Quizás por esta misma razón, las fuentes no detallaron

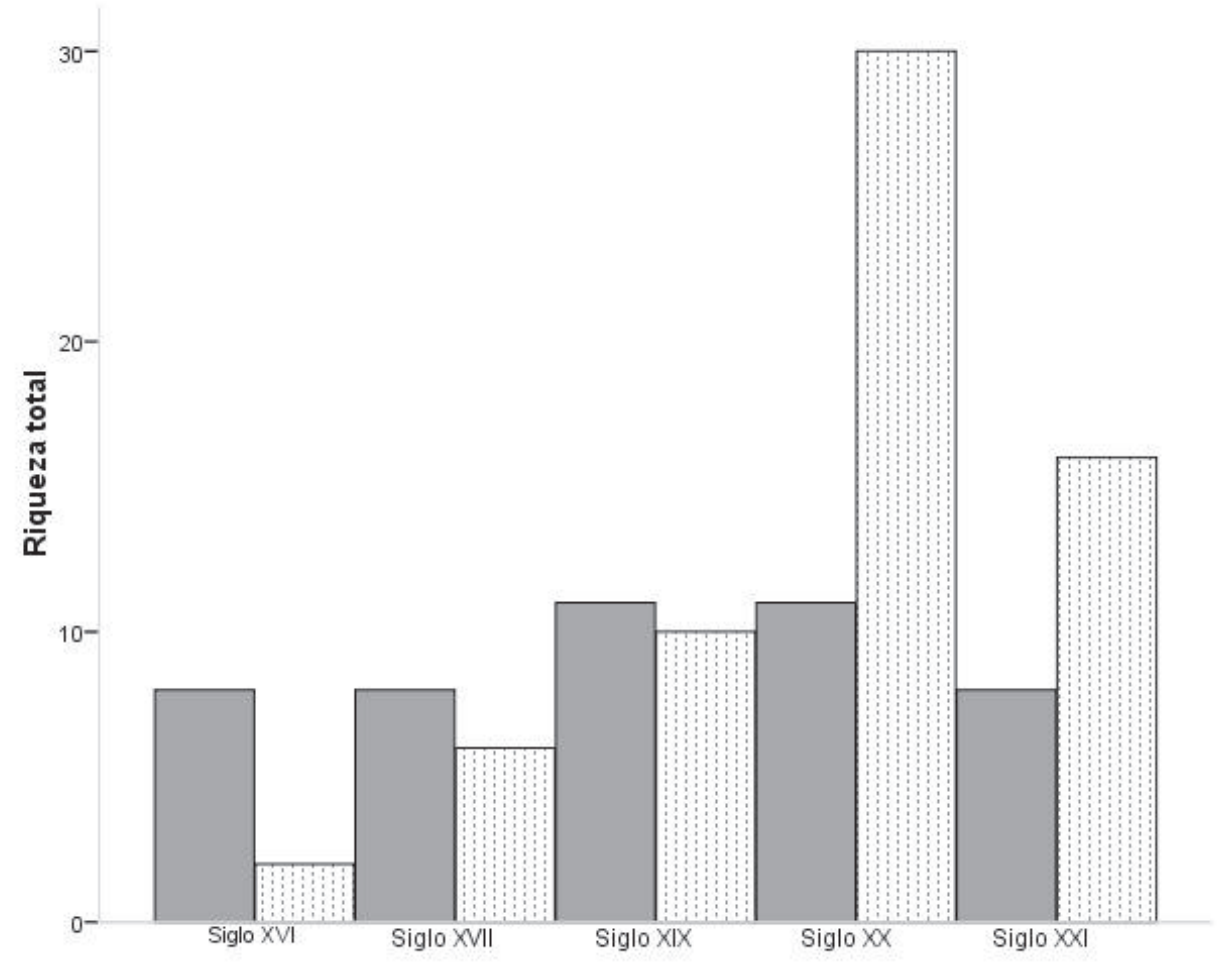

Fig. 3. Número de fuentes revisadas (barras oscuras) y riqueza de POAS comestibles documentadas (barras punteadas) desde el siglo XVI hasta el presente. 
usos locales y siguieron soslayando el uso de recursos vegetales silvestres.

En cambio, a partir del siglo XIX, varios autores de las fuentes poseían formación naturalista, e incluso botánica (ej. Jorge Claraz), haciéndose más evidente el interés por describir las costumbres indígenas, aún sin emplear metodologías rigurosas. Durante los siglos XX y XXI, los trabajos se tornaron cada vez más específicos $\mathrm{y}$ sistemáticos, con objetivos inventariales (Martínez Crovetto, 1968, 1980, 1982), hasta los trabajos etnobotánicos de las últimas décadas que emplean metodologías detalladas (cuali-cuantitativas), indagando no sólo los recursos utilizados, sino también la dinámica de cambio del uso de los mismos en torno a aspectos sociales y ecológicos (Ladio, 2001, 2002, 2006; Ladio \& Lozada 2000; Ochoa \& al., 2010a).

Al comparar la riqueza de POAS encontrada entre siglos (Tabla 3) notamos una muy baja similitud en general, relacionado al hecho de que una gran proporción de especies fue registrada sólo para 1 siglo (Fig. 4). El valor más alto de similitud $(37,5 \%)$ se da entre las especies registradas en las poblaciones rurales estudiadas en este trabajo y las correspondientes a los demás trabajos realizados en comunidades rurales durante el siglo XXI. Si bien, esta baja similitud estaría indicando nuevamente especificidad en las POAS y/o discontinuidades en el uso de estas especies, como señalamos antes, debemos tener presente que muchas especies resultaron invisibles a la documentación. En cambio, unas pocas especies, mostraron cierta continuidad en el registro: Bolax gumifera y Solanum maglia son citadas durante tres siglos; y Alstroemeria aurea y Diposis patagónica se encuentran presentes en las fuentes durante dos siglos. Probablemente estas especies hayan tenido en el pasado un rol distintivo como recurso, y de ahí su registro más intenso. El caso más destacable es Arjona tuberosa que resultó citada en las fuentes a lo largo de 4 siglos, con un registro de utilización que va desde el siglo XVIII hasta los trabajos etnográficos del presente. Ya sea por su amplia distribución, su calidad nutricional y/o su permanencia en las tradiciones orales, esta especie resulta de una importancia cultural única. Finalmente, un aspecto interesante en la dinámica de cambio de la riqueza de especies, es la incorporación de nuevos recursos. Sabemos que los ambientes patagónicos se caracterizan por presentar
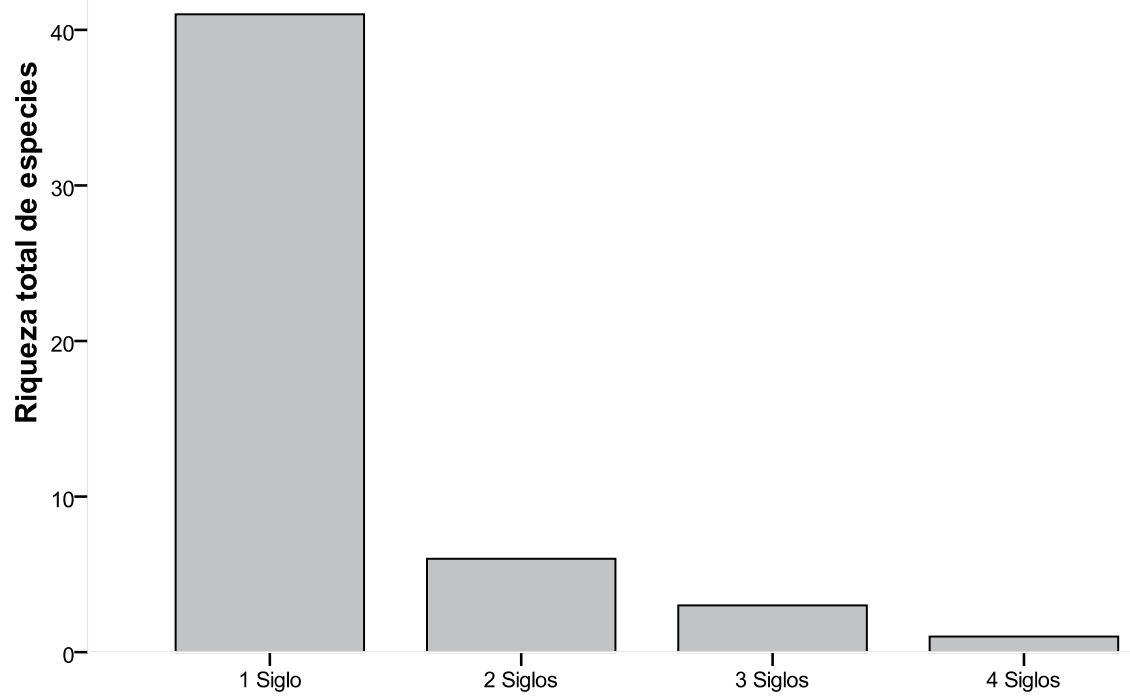

Fig. 4. Continuidad en el registro de las POAS comestibles desde el siglo XVI hasta el presente, en la Patagonia 
un alto porcentaje de plantas introducidas, proceso que probablemente comenzó con la colonización humana de la región durante el Pleistoceno; pero, sin embargo, se estima que hace unos 300 años este flujo se aceleró (Ezcurra \& Brion, 2005). En concordancia con estas ideas, encontramos POAS comestibles exóticas a partir del siglo XIX, con el registro del consumo de las raíces de Hypochaeris radicata y Taraxacum officinale (Martínez Crovetto, 1968), y el ya mencionado caso de Pastinaca sativa para el siglo XXI.

Tabla 1. Plantas con órganos de almacenamiento subterráneos (POAS) registradas en 54 fuentes etnohistóricas y trabajos etnobotánicos en la Patagonia.

\begin{tabular}{|c|c|c|c|c|}
\hline Nombre científico/Familia & Etnoespecie/Fuente & $\begin{array}{l}\text { Unidad } \\
\text { Ecológica }\end{array}$ & Siglo & $\begin{array}{c}\text { Tipo de } \\
\text { órgano } \\
\text { subterráneo }\end{array}$ \\
\hline $\begin{array}{l}\text { Acmella decumbens }(\mathrm{Sm} .) \\
\text { R.K. Jansen/Asteraceae }\end{array}$ & $\begin{array}{l}\text { "ñimñim", “zuma”, "mata diente"/ } \\
\text { Steibel (1997) }\end{array}$ & Ecotono & $\mathrm{XX}$ & Raíz \\
\hline $\begin{array}{l}\text { Adesmia lotoides Hook. f./ } \\
\text { Fabaceae }\end{array}$ & “kiárksh"/ Martínez Crovetto (1968) & Estepa & $\mathrm{XX}$ & Rizoma \\
\hline $\begin{array}{l}\text { Alstroemeria aurea Graham./ } \\
\text { Alstroemeriaceae }\end{array}$ & $\begin{array}{l}\text { “tucuyo"/ Olivares (1878); "tucuyu”, } \\
\text { "tucutucul”/ Menéndez (1896); } \\
\text { "liuto", "amancay", cascabel/ } \\
\text { Martínez Crovetto (1980); } \\
\text { "amancai", "amancay", "liuto", } \\
\text { “cascabel"/ Conticello \& al., (1997) }\end{array}$ & $\begin{array}{l}\text { Bosque } \\
\text { andino- } \\
\text { patagónico }\end{array}$ & XVIII, XX & Rizoma \\
\hline $\begin{array}{l}\text { Alstroemeria ligtu L. / } \\
\text { Alstroemeriaceae }\end{array}$ & $\begin{array}{l}\text { "liltu"/ Machoni (1731); "liuto"/ } \\
\text { Molina (1878) }\end{array}$ & $\begin{array}{l}\text { Bosque } \\
\text { andino- } \\
\text { patagónico }\end{array}$ & XVIII & Rizoma \\
\hline $\begin{array}{l}\text { Alstroemeria patagónica } \text { Phil. } \\
\text { /Alstroemeriaceae }\end{array}$ & “chaquil"/ Ladio (2000) & Estepa & XXI & Rizoma \\
\hline $\begin{array}{l}\text { Alstroemeria pelegrina } \mathrm{L} . / \\
\text { Alstroemeriaceae }\end{array}$ & $\begin{array}{l}\text { "liuto", "peregrina", "rayen cachu”, } \\
\text { "nil"/ Moesbach (1930) }\end{array}$ & $\begin{array}{l}\text { Bosque } \\
\text { andino- } \\
\text { patagónico }\end{array}$ & $\mathrm{XX}$ & Rizoma \\
\hline $\begin{array}{l}\text { Apium prostratum Labill./ } \\
\text { Apiaceae }\end{array}$ & $\begin{array}{l}\text { "apio silvestre", "apio de campo"/ } \\
\text { Ladio \& al. (2007), Ochoa \& al. } \\
\text { (2010b) }\end{array}$ & Ecotono & XXI & Raíz \\
\hline Arctium sp./Asteraceae & "bardana"/ Bourne (1998) & Monte & XIX & Raíz \\
\hline $\begin{array}{l}\text { Arjona patagonica Hombr. \& } \\
\text { Jacq. ex Decne./Schoepfiaceae }\end{array}$ & $\begin{array}{l}\text { "saquel”, "chaquil"/ Martínez } \\
\text { Crovetto (1980) }\end{array}$ & Estepa & $\mathrm{XX}$ & Tubérculo \\
\hline $\begin{array}{l}\text { Arjona tuberosa Cav./ } \\
\text { Schoepfiaceae }\end{array}$ & $\begin{array}{l}\text { "llocon"/ Olivares (1878); "fruta } \\
\text { blanca"/ González (1965); "yahu- } \\
\text { yehuin"/ Cox (1863); "yaukhana"/ } \\
\text { Claraz (1988); "saquel"/ Guinnard } \\
\text { (1947); "macachín", "mata trigo"/ } \\
\text { Conticello \& al. (1997); "loopoñi", } \\
\text { "shakül", "macachín", "chaquil"/ } \\
\text { Steibel (1997); "shaküll"/ Czertock } \\
\text { (1999); "saquel", "chaquil"/ Ladio } \\
\text { (2006); "papita del campo", "papita } \\
\text { del piche", "macachín", "shaquil"/ } \\
\text { Ochoa (trabajo etnográfico actual) }\end{array}$ & $\begin{array}{l}\text { Estepa, } \\
\text { Monte, } \\
\text { Ecotono }\end{array}$ & $\begin{array}{l}\text { XVIII; } \\
\text { XIX, XX; } \\
\text { XXI }\end{array}$ & Tubérculo \\
\hline $\begin{array}{l}\text { Azorella filamentosa Lam./ } \\
\text { Apiaceae }\end{array}$ & $\begin{array}{l}\text { "tésh(ue)n"/ Martinez Crovetto } \\
\text { (1968) }\end{array}$ & Estepa & $\mathrm{XX}$ & Raíz \\
\hline $\begin{array}{l}\text { Azorella lycopodioides } \\
\text { Gaudich./Apiaceae }\end{array}$ & $\begin{array}{l}\text { “tésh(ue)n”, “tesh" / Martínez } \\
\text { Crovetto (1968) }\end{array}$ & Estepa & $\mathrm{XX}$ & Raíz \\
\hline
\end{tabular}




\begin{tabular}{|c|c|c|c|c|}
\hline Nombre científico/Familia & Etnoespecie/Fuente & $\begin{array}{l}\text { Unidad } \\
\text { Ecológica }\end{array}$ & Siglo & $\begin{array}{c}\text { Tipo de } \\
\text { órgano } \\
\text { subterráneo } \\
\end{array}$ \\
\hline $\begin{array}{l}\text { Azorella monantha } \text { Clos./ } \\
\text { Apiaceae }\end{array}$ & $\begin{array}{l}\text { "leña de piedra"/ Sánchez Labrador } \\
\text { (1936), Ochoa (trabajo etnográfico } \\
\text { actual); "tes", "tesh", "tís,h(ue) } \\
\text { n", "tésh(ue)n"/ Martínez Crovetto } \\
\text { (1968); "leña de piedra", "yareta", } \\
\text { "llareta"/ Ladio \& Lozada (2000), } \\
\text { Ladio \& al. (2007), Lozada \& Ladio } \\
\text { (2006) }\end{array}$ & Estepa & $\begin{array}{l}\text { XVIII, } \\
\text { XX, XXI }\end{array}$ & Raíz \\
\hline $\begin{array}{l}\text { Azorella selago Hook. f./ } \\
\text { Apiaceae }\end{array}$ & $\begin{array}{l}\text { "tésh(ue)n", "tísh(ue)n" / Martínez } \\
\text { Crovetto (1968) }\end{array}$ & Estepa & $\mathrm{XX}$ & Raíz \\
\hline $\begin{array}{l}\text { Azorella trifurcata (Gaertn.) } \\
\text { Pers./Apiaceae }\end{array}$ & $\begin{array}{l}\text { "tésh(ue)n", "tísh(ue)n" / Martínez } \\
\text { Crovetto (1968) }\end{array}$ & Estepa & $\mathrm{XX}$ & Raíz \\
\hline $\begin{array}{l}\text { Blumenbachia dissecta (Hook. } \\
\text { \& Arn.) Weigend \& J. Grau/ } \\
\text { Loasaceae }\end{array}$ & $\begin{array}{l}\text { "ortiga blanca", "ortiguilla", "ortiga"/ } \\
\text { Ladio \& Lozada (2000), Ladio } \\
\text { (2006), Ladio \& al. (2007) }\end{array}$ & Estepa & XXI & Raíz \\
\hline $\begin{array}{l}\text { Bolax gummifera (Lam.) / } \\
\text { Apiaceae Spreng. }\end{array}$ & $\begin{array}{l}\text { "capac", "chapac"/ Pigafetta (1941); } \\
\text { "khet-hèla", "gethenn"/ Claraz } \\
\text { (1988); "tus"/ Fitz Roy (1839); } \\
\text { "jaye", "pechero"/ Lista (1894); } \\
\text { "kallfüko"/ Czertock (1999) }\end{array}$ & Estepa & $\begin{array}{l}\text { XVI, XIX, } \\
\text { XX }\end{array}$ & Raíz \\
\hline $\begin{array}{l}\text { Boopis australis Decne./ } \\
\text { Calyceraceae }\end{array}$ & $\begin{array}{l}\text { "so", "sos"/ Gusinde (1931); "íshta"/ } \\
\text { Martínez Crovetto (1968) }\end{array}$ & Estepa & $\mathrm{XX}$ & Raíz \\
\hline $\begin{array}{l}\text { Conanthera bifolia Ruiz \& } \\
\text { Pav./Tecophilaeaceae }\end{array}$ & $\begin{array}{l}\text { "papita de campo", "nao"/ Moesbach } \\
\text { (1930) }\end{array}$ & $\begin{array}{l}\text { Bosque } \\
\text { andino- } \\
\text { Patagónico }\end{array}$ & $\mathrm{XX}$ & Bulbo \\
\hline $\begin{array}{l}\text { Dioscorea gayi Phil./ } \\
\text { Dioscoreaceae }\end{array}$ & "huanqui"/ Moesbach (1930) & $\begin{array}{l}\text { Bosque } \\
\text { andino- } \\
\text { patagónico }\end{array}$ & $\mathrm{XX}$ & Tubérculo \\
\hline $\begin{array}{l}\text { Dioscorea saxatilis Poepp./ } \\
\text { Dioscoreaceae }\end{array}$ & $\begin{array}{l}\text { "huanqui", "huanque", "ranqui", } \\
\text { "mahuida poñu", "papa del monte"/ } \\
\text { Moesbach (1992) }\end{array}$ & $\begin{array}{l}\text { Bosque } \\
\text { andino- } \\
\text { patagónico }\end{array}$ & $\mathrm{XX}$ & Tubérculo \\
\hline $\begin{array}{l}\text { Diposis bulbocastanum DC./ } \\
\text { Apiaceae }\end{array}$ & $\begin{array}{l}\text { "pique - pique" / Jerónimo Vivar } \\
\text { (1966) }\end{array}$ & $\begin{array}{l}\text { Bosque } \\
\text { andino- } \\
\text { patagónico }\end{array}$ & XVI & Tubérculo \\
\hline $\begin{array}{l}\text { Diposis patagónica } \text { Skottsb./ } \\
\text { Apiaceae }\end{array}$ & $\begin{array}{l}\text { "llocon"/ Menéndez (1896), Ochoa } \\
\text { (Trabajo etnográfico actual); } \\
\text { "llokon"/ Czertock (1999); "yocón", } \\
\text { "llucún", "llucón", "llacúm"/ Ladio } \\
\text { \& Lozada (2000), Ladio \& al. (2007), } \\
\text { Lozada \& Ladio (2006) }\end{array}$ & Ecotono & $\begin{array}{l}\text { XVIII, } \\
\text { XX, XXI }\end{array}$ & Tubérculo \\
\hline $\begin{array}{l}\text { Eringyum paniculatum Cav. } \\
\text { \& Dombey ex F. Delaroche/ } \\
\text { Apiaceae }\end{array}$ & $\begin{array}{l}\text { "chupalla", "cardoncillo"/ Ladio } \\
\text { (2006), Lozada \& Ladio (2006) }\end{array}$ & Ecotono & XXI & Rizoma \\
\hline $\begin{array}{l}\text { Gunnera tinctoria Molina) } \\
\text { Mirb./Gunneraceae }\end{array}$ & “pangue"/ Cox (1863) & $\begin{array}{l}\text { Bosque } \\
\text { andino- } \\
\text { patagónico }\end{array}$ & XIX & Raíz \\
\hline $\begin{array}{l}\text { Herbertia lahue (Mol.) } \\
\text { Goldbl./Iridaceae }\end{array}$ & $\begin{array}{l}\text { "lahue", "lahui", "lahuu", "lawii"/ } \\
\text { Mosbach (1991) }\end{array}$ & Ecotono & $\mathrm{XX}$ & Bulbo \\
\hline $\begin{array}{l}\text { Hoffmanggesia erecta } \text { Phil./ } \\
\text { Fabaceae }\end{array}$ & $\begin{array}{l}\text { "porotillo", "pata de choique"/ Ladio } \\
\text { (2006) }\end{array}$ & Ecotono & XXI & Raíz tuberosa \\
\hline $\begin{array}{l}\text { Hoffmannseggia glauca } \\
\text { (Ortega) Eifert/Fabaceae }\end{array}$ & "shakül”, "porotillo"/ Steibel (1997) & Ecotono & $\mathrm{XX}$ & Raíz tuberosa \\
\hline
\end{tabular}




\begin{tabular}{|c|c|c|c|c|}
\hline Nombre científico/Familia & Etnoespecie/Fuente & $\begin{array}{l}\text { Unidad } \\
\text { Ecológica }\end{array}$ & Siglo & $\begin{array}{c}\text { Tipo de } \\
\text { órgano } \\
\text { subterráneo }\end{array}$ \\
\hline $\begin{array}{l}\text { Hypochaeris incana (Hook. \& } \\
\text { Arn.) Macloskie/Asteraceae }\end{array}$ & $\begin{array}{l}\text { “oitá", “oitáí", “oîtá”, “oí’tá”/ } \\
\text { Martínez Crovetto (1968) }\end{array}$ & Estepa & $\mathrm{XX}$ & Raíz \\
\hline $\begin{array}{l}\text { Hypochaeris radicata } \mathrm{L} . / \\
\text { Asteraceae }\end{array}$ & $\begin{array}{l}\text { “oitá”, “oitáí”, “oîtá", “oí’tá”/ } \\
\text { Martínez Crovetto (1968) }\end{array}$ & Ecotono & $\mathrm{XX}$ & Raíz \\
\hline Juncus sp./Juncaceae & $\begin{array}{l}\text { "junco"/ MartínezCrovetto 1980; } \\
\text { Ochoa (trabajo etnográfico actual) }\end{array}$ & Ecotono & XX, XXI & Tubérculo \\
\hline $\begin{array}{l}\text { Lophosoria quadripinnata (J.F. } \\
\text { Gmel.) C. Chr./Dicksoniaceae }\end{array}$ & $\begin{array}{l}\text { "palmita", "ampe", "añpe" / } \\
\text { Moesbach (1930) }\end{array}$ & $\begin{array}{l}\text { Bosque } \\
\text { andino- } \\
\text { Patagónico }\end{array}$ & $\mathrm{XX}$ & Rizoma \\
\hline $\begin{array}{l}\text { Maihueniopsis darwinii var. } \\
\text { hickenii (Hensl.) F. Ritter/ } \\
\text { Cactaceae }\end{array}$ & $\begin{array}{l}\text { “chupasangre” / Ladio \& Lozada } \\
\text { (2000), Ladio (2006), Lozada \& } \\
\text { Ladio (2006) }\end{array}$ & Estepa & XXI & Raíz tuberosa \\
\hline $\begin{array}{l}\text { Oreomyrrhiz andicola (Kunth) } \\
\text { Hook. f./Apiaceae }\end{array}$ & “seltái” / Martínez Crovetto (1968) & Estepa & XX & Raíz \\
\hline $\begin{array}{l}\text { Osmorhiza chilensis Hook. \& } \\
\text { Arn./Apiaceae }\end{array}$ & $\begin{array}{l}\text { "amor seco"/ Martínez-Crovetto } \\
\text { (1980); "cacho de cabra", "quin } \\
\text { quin", "ñonquín"/ Conticello \& al. } \\
\text { (1997) }\end{array}$ & $\begin{array}{l}\text { Bosque } \\
\text { andino- } \\
\text { patagónico }\end{array}$ & $\mathrm{XX}$ & Raíz \\
\hline $\begin{array}{l}\text { Oxalis adenophylla Gillies ex } \\
\text { Hook. \& Arn./Oxalidaceae }\end{array}$ & $\begin{array}{l}\text { "cuye colorado", "cuye bueno"/ } \\
\text { Ladio \& Lozada (2000), Ladio } \\
\text { (2006), Lozada \& Ladio (2006), } \\
\text { Ochoa \& al. (2010b); "cuye"/ Ochoa } \\
\text { (Trabajo etnográfico actual) }\end{array}$ & Ecotono & XXI & Rizoma \\
\hline $\begin{array}{l}\text { Oxalis enneaphylla } \text { Cav./ } \\
\text { Oxalidaceae }\end{array}$ & $\begin{array}{l}\text { âl haístikin/ Martínez-Crovetto } \\
\text { (1980) }\end{array}$ & Estepa & $\mathrm{XX}$ & Rizoma \\
\hline $\begin{array}{l}\text { Oxalis nahuelhuapensis Speg./ } \\
\text { Oxalidaceae }\end{array}$ & cuye/ Ochoa \& al. (2010a) & Ecotono & XXI & Raíz \\
\hline Pastinaca sativa L./Apiaceae & $\begin{array}{l}\text { "pana"/ Ochoa (Trabajo etnográfico } \\
\text { actual) }\end{array}$ & Ecotono & XXI & Raíz \\
\hline $\begin{array}{l}\text { Phragmites australis (Cav.) } \\
\text { Trin. ex Steud./Poaceae }\end{array}$ & $\begin{array}{l}\text { “carrizo” /Ladio (2006); Ladio \& al. } \\
\text { (2007) }\end{array}$ & Ecotono & XXI & Rizoma \\
\hline $\begin{array}{l}\text { Prosopanche bonacine Speg./ } \\
\text { Hydnoraceae }\end{array}$ & $\begin{array}{l}\text { "poñi, poñu”/ Guinnard (1947); } \\
\text { "mamül poñi”, "papa del monte"/ } \\
\text { Steibel (1997) }\end{array}$ & Estepa & XIX & Raíz \\
\hline $\begin{array}{l}\text { Rhodophiala elwesii (C.H. } \\
\text { Wright) Traub }\end{array}$ & $\begin{array}{l}\text { "amancay"/ Martínez Crovetto } \\
\text { (1980); "amancay, "ajo silvestre", } \\
\text { "cebolleta", "ajo del diablo"/ Ladio } \\
\text { (2006), Ladio \& al. (2007) }\end{array}$ & Estepa & XXI & Bulbo \\
\hline $\begin{array}{l}\text { Schoenoplectus californicus } \\
\text { (C.A. Mey.) Soják var. } \\
\text { californicus/Juncaceae }\end{array}$ & “junco", “salma”/ Guinnard (1947) & Estepa & XIX & Raíz \\
\hline $\begin{array}{l}\text { Solanum maglia Schltdl. / } \\
\text { Solanaceae }\end{array}$ & $\begin{array}{l}\text { "pomas de tierra", "maglia"/ Molina } \\
\text { (1878); "akina, papa silvestre"/ } \\
\text { Darwin (1964); "malle"/ Moesbach } \\
\text { (1930); "malla", "aquina"/ Cardenas } \\
\text { \& al. (1999) }\end{array}$ & Ecotono & $\begin{array}{l}\text { XVIII; } \\
\text { XIX, XX }\end{array}$ & Tubérculo \\
\hline $\begin{array}{l}\text { Sysirinchium striatum } \mathrm{Sm} . / \\
\text { Iridaceae }\end{array}$ & “illmu”/ Molina (1782) & $\begin{array}{l}\text { Bosque } \\
\text { andino- } \\
\text { patagónico }\end{array}$ & XVIII & Rizoma \\
\hline $\begin{array}{l}\text { Taraxacum gilliesi Hook. \& } \\
\text { Arn. }\end{array}$ & $\begin{array}{l}\text { “oitá”, “oitáí”, “oîtá”, “oí’tá”/ } \\
\text { Martínez Crovetto (1968) }\end{array}$ & Estepa & $\mathrm{XX}$ & Raíz \\
\hline
\end{tabular}




\begin{tabular}{|c|c|c|c|c|}
\hline Nombre científico/Familia & Etnoespecie/Fuente & $\begin{array}{l}\text { Unidad } \\
\text { Ecológica }\end{array}$ & Siglo & $\begin{array}{c}\text { Tipo de } \\
\text { órgano } \\
\text { subterráneo }\end{array}$ \\
\hline $\begin{array}{l}\text { Taraxacum officinale G. Weber } \\
\text { ex F.H. Wigg. }\end{array}$ & $\begin{array}{l}\text { “oitá”, “oitáí”, “oîtá”, oí’tá/ Martínez } \\
\text { Crovetto (1968) }\end{array}$ & Ecotono & $\mathrm{XX}$ & Raíz \\
\hline $\begin{array}{l}\text { Tristagma patagonicum } \\
\text { (Baker) Traub./Alliaceae }\end{array}$ & $\begin{array}{l}\text { "chaleo" / Ladio (2006); "cebollita de } \\
\text { campo"/ Ochoa (Trabajo etnográfico } \\
\text { actual) }\end{array}$ & Estepa & XXI & Bulbo \\
\hline $\begin{array}{l}\text { Tropaeolum patagonicum } \\
\text { Speg./Tropaeolaceae }\end{array}$ & $\begin{array}{l}\text { “yalemska", "yalemtska"/ (Claraz } \\
\text { 1988); “chala"/ Fitz Roy (1839); } \\
\text { "yaulëmsk" /Sánchez Labrador } \\
\text { (1936); "chalia"/ Martínez Crovetto } \\
\text { (1982) }\end{array}$ & Ecotono & $\begin{array}{l}\text { XVIII, } \\
\text { XIX }\end{array}$ & Tubérculo \\
\hline $\begin{array}{l}\text { Tropaeolum porifolium (Cav.) } \\
\text { L.Andersson \& S.Andersson/ } \\
\text { Tropaeolaceae }\end{array}$ & $\begin{array}{l}\text { "yahtscha"/ Claraz (1988) ; "tchali"/ } \\
\text { Vaulx (1901); "chali" / Moreno } \\
\text { (1969); “curuhuilla"/ Ladio \& Lozada } \\
\text { (2004), Ladio (2006); "chaguala", } \\
\text { "papita colorada", "batata"/ Ochoa } \\
\text { (trabajo etnográfico actual) }\end{array}$ & Ecotono & XIX, XXI & Tubérculo \\
\hline Typha spp./Typhaceae & “espadaña”/ Musters (1997) & Estepa & XIX & Raíz \\
\hline
\end{tabular}

Tabla 2. Etnoespecies no determinadas taxonómicamente en la revisión bibliográfica sobre plantas con órganos de almacenamiento subterráneos comestibles presentes en la Patagonia.

\begin{tabular}{|c|c|c|c|}
\hline Nombre vulgar & Fuente & Unidad Ecológica & Siglo \\
\hline "achupallas" & Jerónimo Vivar (1966) & $\begin{array}{l}\text { Bosque andino- } \\
\text { patagónico }\end{array}$ & XVI \\
\hline "pihui" & $\begin{array}{l}\text { Olivares (1878); } \\
\text { Menéndez (1896) }\end{array}$ & $\begin{array}{l}\text { Bosque andino- } \\
\text { patagónico }\end{array}$ & XVIII \\
\hline "mutiti" & Menéndez (1896) & $\begin{array}{l}\text { Bosque andino- } \\
\text { patagónico }\end{array}$ & XVIII \\
\hline "tes" & González (1965) & Estepa & XVIII \\
\hline "cocolla" & Claraz (1988) & Monte & XVIII \\
\hline "huispi", "wispi" & Claraz (1988) & Monte & XVIII \\
\hline "peya" & Claraz (1988) & Monte & XVIII \\
\hline "appeley", "papa silvestres" & Musters (1997) & Estepa & XIX \\
\hline "kerupe", "kurpe" & Moreno (1969) & Estepa & XIX \\
\hline "churriaca", "panchurriaca" & San Martin (1919) & Ecotono & $\mathrm{XX}$ \\
\hline "collocho", "coyocho" & San Martin (1919) & Ecotono & $\mathrm{XX}$ \\
\hline "shaalt" & Gallardo (1910) & Ecotono & $\mathrm{XX}$ \\
\hline "kushiwill" & Czertock (1999) & Estepa & $\mathrm{XX}$ \\
\hline "chaleu" & Czertock (1999) & Estepa & $\mathrm{XX}$ \\
\hline "pichorca" & Ladio \& Lozada (2000) & Estepa & $\mathrm{XX}$ \\
\hline "como la sandia" & $\begin{array}{l}\text { Ochoa (Trabajo } \\
\text { etnográfico actual) }\end{array}$ & Estepa & XXI \\
\hline "ocre" & $\begin{array}{l}\text { Ochoa (Trabajo } \\
\text { etnográfico actual) }\end{array}$ & Estepa & XXI \\
\hline
\end{tabular}


BONPLANDIA 20(2). 2011

Tabla 3. Matriz de Similitud de Jaccard que compara la riqueza de especies registradas entre las fuentes de los siglos XVI hasta el presente.

\begin{tabular}{lcccccc}
\hline & Siglo XVI & $\begin{array}{c}\text { siglo } \\
\text { XVIII }\end{array}$ & siglo XIX & siglo XX & siglo XXI & $\begin{array}{c}\text { POAS en registradas } \\
\text { en las 4 comunidades } \\
\text { rurales estudiadas }^{2}\end{array}$ \\
\hline Siglo XVI & 1 & 0 & 0 & 0 & 0 & 0 \\
Siglo XVIII & 0 & 1 & 0,133 & 0,121 & 0 & 0,167 \\
siglo XIX & 0 & 0,133 & 1 & 0,135 & 0,087 & 0,188 \\
Siglo XX & 0 & 0,121 & 0,135 & 1 & 0,098 & 0,083 \\
$\begin{array}{l}\text { Siglo XXI } \\
\text { POAS en }\end{array}$ & 0 & 0 & 0,087 & 0,098 & 1 & $\mathbf{0 , 3 7 5}$ \\
$\begin{array}{l}\text { registradas en las 4 } \\
\text { comunidades rurales }\end{array}$ & 0 & 0,167 & 0,188 & 0,083 & 0,375 & 1 \\
estudiadas $^{2}$ & & & & & & \\
\hline
\end{tabular}

${ }^{1}$ En este conjunto de datos no se incluyen las del trabajo etnográfico en las comunidades de Villa Lanquín, Lagunita Salada, El Escorial y Nahuel Pan.

${ }^{2}$ Corresponden a la riqueza total relevada en las comunidades de Villa Llanquín, Lagunita, El Escorial y Nahuel Pan.

\section{Conclusiones}

A pesar de las limitaciones que posee el trabajo con fuentes históricas dispersas, fragmentarias y de objetivos diversos como sucede en el registro patagónico, su análisis nos permite ampliar nuestra mirada sobre la utilización de los recursos naturales a lo largo del tiempo, contextualizando los hallazgos no sólo en función de razones ecológicoambientales, sino también socio-económicas y geopolíticas. Los resultados obtenidos indican que las plantas con órganos de almacenamiento subterráneos comestibles han tenido un valor utilitario muy relevante en el pasado, que esperamos pueda ser activado en el presente dado que forma parte del legado cultural de nuestros pueblos patagónicos. Estos recursos han sido ignorados y abandonados por las distintas sociedades, pero constituyen una cosecha "oculta" de la Patagonia con gran potencialidad biocultural.

\section{Bibliografía}

ALBUQUERQUE, U. P., \& R. F. PAIVA DE LUCENA. 2005. Can apparency affect the use of plants by local people in the tropical forests?. Interciencia Vol. $30 \mathrm{~N}^{\mathrm{o}}$ 8: 506-511.

ALBUQUERQUE, U. P., PAIVA DE LUCENA, R. F.
\& L. V- F. CRUZ DA CUNHA. 2010. Métodos e técnicas na pesquisa Etnobiológica y Etnoecológica (1st Edn), Nuppea, Recife, Brasil.

AGRESTI, A. 1996. An Introduction to Categorical Data Analysis. John Wiley \& Sons. New York.

BANDIERI, S. 2005. Historia de la Patagonia. Editorial Sudamericana. Buenos Aires. 445 pp.

BEGON, M., TOWNSEND, C. R. \& J. L. HARPER. 2005. Ecology: From Individuals to Ecosystems, 4th edn. Blackwell Science, Oxford. 752 pp.

BERKES, F. \& C. FOLKE. 2002. Back to the future: ecosystem dynamics and local knowledge. Pages 121-146 in L. H. Gunderson and C. S. Holling, editors. Panarchy: understanding transformations in human and natural systems. Island Press, Washington, D.C., USA. 448 pp.

BOURNE, B. F. 1998. Cautivo en la Patagonia. Emecé Editores. Buenos Aires. 217 pp.

CABRERA, L. A. 1976. Enciclopedia Argentina de Agricultura y Ganadería. Regiones Fitogeográficas Argentinas. ACME S.A.C.I. Buenos Aires. 85 pp.

CAIN, N., DARBYSHIRE, S. J., FRANCIS, A., NURSE, R. E.\& M. J. SIMARD. 2010. The Biology of Canadian weeds. 144. Pastinaca sativa L. Can. J. Plant Sci. 90: 217-240.

CAMPBELL, A. 1986. The use of wild food plants, and drought in Botswana. Journal of Arid Environments, 11: 81-91.

CHAPMAN, A. 1986. Los selk'nam. La vida de los onas. 1982. Ediciones Emecé Buenos Aires. 287 pp.

CLARAZ, J. 1988. Diario de viaje de exploración al Chubut. 1865-1866. Ediciones Marymar, Buenos Aires. $189 \mathrm{pp}$.

CONTICELLO, L., GANDULLO, R., BUSTAMANTE, 
A. \& C. TARTAGLIA. 1997. El uso de las plantas medicinales por la comunidad mapuche de San Martín de los Andes, Prov. de Neuquén (Argentina). Parodiana 10 (1-2): 165-180.

CORREA, M. N. (ed.). 1969-1999. Flora Patagónica. Colec. Ci. Inst. Nac. Tecnol. Agropecu. 8, Partes $1-7$.

COX, G. E. 1863 Viaje en las rejiones septentrionales de la Patagonia 1862-63. Imprenta Nacional. Santiago de Chile. 273 pp.

CRUZ, L. DE LA [1806] 1969. Viaje a su costa del Alcalde provincial del muy ilustre Cabildo de la Concepción de Chile. Desde el fuerte de Ballenar... hasta la ciudad de Buenos Aires... En: de Angelis, Pedro (comp.); colección de obras y documentos: 45-389. Buenos Aires, Plus Ultra.

CRUZ CRUZ, J. 1991. Alimentación y Cultura: Antropología de la conducta alimentaria. Ediciones Universidad De Navarra. EUNSA. 293 pp.

CZERTOCK, V. 1999. Historia de vida y memorias de un antiguo. Textos ameghinianos, Biblioteca de la Fundación Ameghino. 110 pp.

DARWIN, C. 1964. Viaje de un naturalista alrededor del mundo (1832-36). Editorial El Ateneo, Buenos Aires. 330 pp.

EYSSARTIER, C., LADIO, A. H. \& M. LOZADA. 2011. Traditional horticultural knowledge change in a rural population of the Patagonian steppe. Journal of Arid Environments 75: 78-86.

EZCURRA, C. \&C. BRION. 2005. Plantas del Nahuel Huapí. Catálogo de la Flora Vascular del Parque Nacional Nahuel Huapí, Argentina. Universidad Nacional del Comahue, Red Latinoamericana de Botánica, S.C. de Bariloche, Argentina. 70 pp.

FALKNER, T. 1911. Descripción de la Patagonia. Universidad Nacional de La Plata. Biblioteca Centenaria, Tomo I. Buenos Aires. 392 pp.

FERNÁNDEZ DE OVIEDO \& VALDÉS, G. 1944. Historia general y natural de las Indias. Editorial Guarania, Asunción del Paraguay.

FERNÁNDEZ, M. \& E. CRIVELLI. 2006. Cambios en la alimentación, las tecnologías y el simbolismo entre los indígenas históricos de la cuenca del río Limay. Estudios de Arqueología Histórica. Investigaciones argentinas Pluridisciplinarias. Museo de la Ciudad de Río Grande, Tierra del Fuego. A. Tapia, M. Ramos y C. Baldassarre, Eds. pp 77-96.

FITZROY, R. 1839. Proceedings of the second expedition, 1831-1836, under the command of Captain..., R.N.,en Narrative of the surveying voyages of his majesty's ships Adventure and Beagle, between the years 1826 and 1836, describing their examination of the southerns shores of South America, and the Beagle's circunnavigation of the globe, II, Londres.

GALLARDO, C. 1910. Los Onas. Buenos Aires: Cabaut
\& Cia. $1^{\circ}$ reimpresión, Ushuaia: Zagier \& Urruty. $395 \mathrm{pp}$.

GÓNGORA DE MARMOLEJO, A. 1990. Historia de todas las cosas que han acaecido en el Reino de Chile y de los que lo han gobernado (1536-1575). Santiago: Ediciones de la Universidad de Chile. $321 \mathrm{pp}$.

GONZÁLEZ DE NÁJERA, A. [1614] 1889. Desengaño y reparo de la guerra del Reino de Chile: donde se manifiestan las principales ventajas que en ella tienen los indios a nuestros españoles ... dividido en cinco partes en que se muestran bárbaros dichos, hechos, casos y usanzas notables ... Santiago de Chile : Impr. Ercilla. 317 pp.

GONZÁLEZ, F. 1965. Diario del viaje que hizo por tierra de Puerto Deseado al Río Negro. 1798. Biblioteca de la Academia Nacional de la Historia, XXIII. Cronistas y viajeros del Río de la Plata, Tomo II. Buenos Aires. 124 pp.

GUINNARD. A. 1947. Tres Años de esclavitud entre los patagones; relato de mi cautiverio, $3^{\circ}$ edición, Bs As, Espasa Calpe. 168 pp.

GUMUCIO, J. C. \& V. INSUNZA. 1992. Las plantas de los mitos y la magia de Chiloé. Boletín del Museo de Historia Natural 43: 9-27.

GUSINDE, M. 1917. Medicina e Higiene de los Araucanos.Tomo I. Instituto de Antropología y Etnografía del Museo de Chile. Santiago de Chile. $18 \mathrm{pp}$.

1931. Die Feuerland-Indianer. Band I. Die Selk'nam. Mödling bei Wien: Anthropos. (Traducción española: Los indios de Tierra del Fuego. Los selk'nam. Buenos Aires: Centro Argentino de Etnología Americana, 1982). 784 pp.

HAVESTADT, B. [1752] 1930. Viaje de fray Francisco Havestadt al Neuquén. En: San Martín, Félix; Neuquén: 215-57. Buenos Aires, Biblioteca del Suboficial.

HLADIK, A., BAHUCHET, C., DUCATILLION, C. \& C. M., HLADIK. 1984. Les plantes a' tubercules de la fore`t dense d'AfriqueCentrale. Revued' Ecologie: La Terre et la Vie, 39, 249-290.

HERNÁNDEZ, T. 1950. Declaración que De orden del Virrei del Perú D. Francisco de Borja, Príncipe de Esquilache, hizo, ante Escribano,...De lo sucedido en las Dos Poblaciones Fundadas en el Estrecho de Magallanes por Pedro Sarmiento de Gambóa. En Embon, Aron.

LAGUNA, F. [1703] 1930. Carta al Superior de la Nueva misión de la compañía de Jesús en Sudamérica en Nahuel Huapi hacia el estrecho de Magallanes. El Río 28 de la Plata visto por viajeros alemanes del siglo XVIII. Revista del Instituto geográfico del Uruguay. 7. 231-235. Montevideo.

LADIO, A. H. 2001. The maintenance of wild edible plant gathering in a Mapuche community of Patagonia. Economic Botany 55 (2): 243-254. 
2002. Las plantas comestibles en el noroeste patagónico y su utilización por las poblaciones humanas: una aproximación cuantitativa. Tesis para optar al grado de Doctor en biología. Universidad Nacional del Comahue. Bariloche. 271 pp.

2006. Uso y conservación de plantas silvestres con órganos subterráneos comestibles en comunidades Mapuche de la estepa patagónica argentina. In: Alburquerque, U.P., Andrade Maris, J.F., Almeida, C.B.R. (Eds.), Tópicos em conservação e etnobotánica de plantas comestíveis. Recife, Brasil, pp. 53-72.

. 2011a. Underexploited wild plant foods of North-Western Patagonia. Rosana Filip (Ed.). Multidisciplinary Approaches on Food Science and Nutrition for the XXI Century. Transworld Research Network. India. 1-16 pp.

- 2011b. Traditional knowledge of edible wild native and exotic plants in the context of cultural change in human populations of arid Patagonia. Bioremediation, Biodiversity and Bioaviability 5 (11): aceptado.

LADIO, A. H. \& M. LOZADA. 2000. Edible wild plant use in a Mapuche community of northwestern Patagonia. Human Ecology 28 (1): 53-71.

. 2004. Patterns of use and knowledge of wild edible plants in distinct ecological environments: a case study of mapuche community from northwestern Patagonia. Biodiversity and Conservation 13: 11531173.

LADIO, A. H., LOZADA, M. \& M. WEIGANDT. 2007. Comparison of traditional wild plant knowledge between aboriginal communities inhabiting arid and forest environments in Patagonia, Argentina. Journal of Arid Environments 69: 695-715.

LATCHAM, R. E. 1929. La Leyenda de Los Césares: su origen y evolución. Imp. Cervantes. Santiago. $254 \mathrm{pp}$.

LISTA, R. 1894. Una raza que desaparece. Los indios Tehuelches. Buenos Aires. 85 pp.

LOZADA, M., M. WEIGANDT \& A. H. LADIO. 2006. Cultural Transmission of Ethnobotanical Knowledge in a Rural Community of Northwestern Patagonia, Argentina. Economic Botany 60(4): 374-385.

MACHONI, A. 1731. Las siete estrellas de la mano de Jesús, Por el Padre Antonio Machoni de la Compañía de Jesús, natural de Cerdeña, Recor del Colegio Máximo de Córdoba del Tucumán y Procurador General à Roma por su Provincia del Paraguay. Córdoba, Colegio de la Assumpcion, Joseph Santos Balbàs. 387 pp.

MARIÑO DE LOBERA, P. [1528-1595] 1861. Crónica del Reino de Chile. Colección de historiadores de Chile y de documentos relativos a la historia nacional. Santiago: Imprenta del Ferrocarril. 465 pp.

MARLOWE, F. W. \& J. C. BERBESQUE. 2009.
Tubers as fallback foods and their impact on Hadza hunter-gatherers. American Journal of Physical Anthropology 140: 151-158.

MARTÍNEZ CROVETTO, R. 1968. Nombres de plantas y su utilidad según los indios Araucanos Pampas del Oeste de Buenos Aires. Estudios etnobotánicos III. Etnobiológica 12: 1-24.

1980. Apuntes sobre la vegetación de los Alrededores del Lago Cholila. Publicación Técnica. 1: 1-22.

1982. Breve panorama de las plantas utilizadas por los indios de Patagonia y Tierra del Fuego. Suplemento Antropológico. Universidad Católica de Asunción 17(1): 61-97.

MARTINIC, B. M. 2006. Historia de la Región de Magallanes. Ediciones de la Universidad de Magallanes.

MAYNE, R. 1874. Derrotero del Estrecho de Magallanes y de los Canales que conducen al Golfo de Peñas según los últimos trabajos del Capitán ... Richard C. Mayne, publicados en 1871 por el Almirantazgo inglés : ampliado en la parte concerniente a los Canales interiores de la Tierra del Fuego con datos españoles ... Madrid : Dirección de Hidrografía. 112 pp.

MEDEIROS, M. F. T. 2009. Etnobotânica Histórica: Princípios e Procedimientos. NUPEEA/Sociedad Brasileira de Etnobiología e Etnoecología, Recife. $83 \mathrm{pp}$.

MENÉNDEZ, F. [1791-1794] 1896. Diario del P. Fr. Francisco Menéndez, Predicador General. Valparaíso, Niemeyer. 152 pp.

MOESBACH, E. W. 1930. Vida y costumbres de los indígenas araucanos en la segunda mitad del siglo XIX. Imprenta Universitaria Estado 63. Santiago de Chile. 464 pp.

_ 1992. Botánica Indígena de Chile. Museo Chileno de Arte Precolombino -Fundación Andes Editorial Andrés Bello. Chile. 140 pp.

MOLINA, J. I. [1795] 1878 Historia natural y civil de Chile. Libro IV. MEDINA; J. Colección de Historiadores de Chile y Documentos relativos a la historia nacional. T XI, Santiago. Mercurio. 382 pp.

MORENO, F. P. 1969. Viaje a la Patagonia Austral. 1876-1877. Solar-Hachette. Buenos Aires, 407 pp.

MORI, J. D. 1889. Relación hecha por...de la expedición de Simón de Alcazaba al estrecho de Magallanes desde que salió de Sanlúcar de Barrameda en veinte $\mathrm{y}$ uno de Setiembre de mil quinientos treinta $\mathrm{y}$ cuatro hasta que llegó a Santo Domingo en Agosto de mil quinientos treinta y cinco; dirigida a un amigo suyo de Sevilla, 20 de Octubre de 1535. En J.T. Medina, Colección de documentos inéditos para la historia de Chile desde el viaje de Magallanes hasta la batalla de Maipo 1518-1818, III. Santiago de Chile. 315 pp.

MORENO, J. O. \& B. VIDELA. 2008. Rastreando ausencias: la hipótesis del abandono del uso de 
los recursos marinos en el momento ecuestre en la Patagonia continental. Magallania, 36 (2): 91-104, Punta Arenas, Chile

MUELLER-DOMBOIS, D. \& H. ELLENBERG. 1974. Aims and methods of vegetation ecology. John Wiley. New York. 547 pp.

MUSTERS, G. C. 1997. Vida entre los Patagones. El Elefante Blanco. Buenos Aires, 375 pp.

NAVARRO FLORIA, P. 2006. Paisajes del progreso. La Norpatagonia en el discurso científico y político argentino de fines del siglo XIX y principios del XX. Scripta Nova: Revista electrónica de geografía y ciencias sociales, $\mathrm{N}^{\mathrm{o}}$. Extra 10, $218 \mathrm{pp}$.

NICOLETTI, M. A. 2004. La conflictiva incorporación de la Patagonia como tierra de misión (1879-1907), Boletín Americanista, $\mathrm{N}^{\circ}$ 54, Barcelona, pp. 145167.

NOCCETI, O \& L. B. MIR. 2000. Relaciones de la jornada a los Césares de 1625. Introducción, estudio preliminar y transcripción paleográfica, Universidad Nacional de Quilmes, Ediciones Amerindia, Argentina. 152 pp.

NOY-MEIR, I. 1978. Desert Ecosystems: Environment and producers. Annual Review of Ecology and Systematics 4:25-52.

NÚÑEZ DE PINEDA Y BASCUÑÁN, F. 1861. Cautiverio feliz, y razón de las guerras dilatadas de Chile. Colección de historiadores de Chile y de documentos relativos a la historia nacional. Santiago: Impr. del Ferrocarril. 546 pp.

OCHOA, J. J. \& H. LADIO. 2010. Plantas con órganos subterráneos en la Patagonia Argentina: patrones etnobotánicos y biogeográficos. X Congreso Latinoamericano de Botánica. 4 al 10 de Octubre de 2010 La Serena, Chile.

OCHOA, J. J., LADIO, A. H. \& M. LOZADA. 2010a. Aspectos ecológicos y socioculturales asociados al uso de plantas silvestres en la población rural de Arroyo las Minas (Río Negro, Argentina), pp: 457-465. En: Tradiciones y Transformaciones en Etnobotánica. Pochettino ML, Ladio AH, Arenas (eds).

- 2010b. Uso de recursos herbolarios entre mapuches y criollos de la comunidad campesina de Arroyo Las Minas (Río Negro, Patagonia Argentina). Boletín Latinoamericano y del Caribe de Plantas Medicinales y Aromáticas, 9 (4), 269-276.

ODEA, K. 1991. Traditional diet and food preferences of Australian aboriginal hunter-gatherers. Philos Trans R Soc Lond Biol Sci 334:233-241.

OLIVARES, M. DE. 1878. Historia de la Compañía de Jesús en Chile (1593-1736). Colección de Historiadores de Chile y documentos relativos a la Historia Nacional. T VII. Santiago de Chile, Mercurio. 508 pp.

ORBIGNY, A. D. d'.1945. Viaje a la América Meridional: Brasil, República del Uruguay, República Argentina,
La Patagonia, República de Chile, República de Bolivia, República del Perú: realizado de 1826 a 1833. Tomo 1, 2, 3, 4. Bs As.

OVALLE, A. 1646. Histórica relación del Reyno de Chile, y de las missiones, y ministerios que exercita en él la Compañía de Jesús, VIII. Roma. 455 pp.

PETERS, C. R. \& E. M. OBRIEN. 1981. The early hominid plant-food niche-insights from an analysis of plant exploitation by Homo, Pan, and Papio in eastern and southern-Africa. Curr Anthropol 22:127-140.

PETERS, C.R., OBRIEN, E. M. \& E. O. BOX. 1984. Plant types and seasonality of wild-plant foods, Tanzania to Southwestern Africa: Resources for models of the natural environment. Journal of Human Evolution Vol 13, Issues 5: 397-414.

PHILLIPS, O. \& A. H. GENTRY. 1993. The useful plants of Tambopata, Perú: I. Statistical hypothesis tests with a new quantitative technique. Econ. Bot. 47, pp: 15-32.

PIGAFETTA, A. 1941. Primer viaje en torno del globo. Buenos Aires-México, Espasa-Calpe Argentina, S.A. 192 pp.

ROSALES, D. 1877. Historia general del reyno de Chile. Flandes indiano. Valparaíso, Imprenta del Mercurio; 3 tomos.

SÁNCHEZ LABRADOR, J. [1772] 1936. Los indios pampas, puelches, patagones. Buenos Aires, Viau y Zona. 255 pp.

SAN MARTIN, F. 1919. Neuquén. Fondo Editorial Neuquino. 246 pp.

SCHOBINGER, J. 1958-1959. Conquistadores, misioneros y exploradores del Neuquén. Antecedentes para el conocimiento etnográfico del noroeste patagónico. Runa IX (1-2):107-123. Buenos Aires.

SEÑORET, M. 1896. Memoria del Gobernador de Magallanes : la tierra del fuego i sus naturales. Santiago de Chile, Imprenta Nacional. 44 pp.

STEIBEL, P. E. 1997. Nombres y usos de las plantas aplicados por los Indios Ranqueles de La Pampa (Argentina). Rev Fac Agron Univ Nac La Pampa 9: 14-15.

TORREJÓN, F. \& M. CISTERNAS. 2002. Alteraciones del paisaje ecológico araucano por la asimilación mapuche de la agroganadería hispano-mediterránea (siglos XVI y XVII). Revista Chilena de Historia Natural 75: 729-736.

VAULX, H. DE LA. 1901. Voyage en Patagonie. Paris. $173 \mathrm{pp}$.

VIEDMA, A. 1837. Diario de un viaje a la costa de la Patagonia, para reconocer los puntos en donde establecer poblaciones con una descripción de la naturaleza de los terrenos, de sus producciones y habitantes; desde el puerto de Santa Elena hasta la boca del Estrecho de Magallanes. Imprenta del Estado. Buenos Aires. 128 pp. 
VEHEDOR, A. 1866. Relación de las cosas que sucedieron en la armada de Simón de Alcazaba. En Luis Torres de Mendoza, Colección de documentos inéditos, relativos al descubrimiento, conquista y organización de las antiguas poseciones españolas en América y Oceanía, V. Madrid.

VIGNATI, M. A. 1939. Los indios poyas. Notas del Museo de La Plata, 4. Antropología 12: 211-44. Buenos Aires.

—. 1941. Contribución a la etnobotánica indígena. El 'pan' de los Patagones protohistóricos. Notas del Museo de La Plata, Tomo VI, Antropología 23: 321336. La Plata.

1963. CARTA RELACIÓN. MASCARDI, N. (1670). Antecedentes para la protoetnografía del Norte de la Patagonia. Boletín de la Academia Nacional de la Historia, 34/2. 495-528. Buenos Aires. Academia Nacional de la Historia.
VILLAGRÁN, C. 1998. Etnobotánica indígena de los bosques de Chiles. Sistema de clasificación de un recurso de uso múltiple. Revista Chilena de Historia Natural 71: 245-268.

VILLARINO, B. 1972. Diario del piloto de la real Armada Don Basilio Villarino del reconocimiento que hizo del río Negro en la costa oriental de Patagonia en el año de 1782. En De Angelis, Pedro (comp.) Colección de obras y documentos: 9671138. Buenos Aires, Plus Ultra.

VINCENT, A. S. 1985. Plant foods in savanna environments: a preliminary report of tubers eaten by the Hadza of northern Tanzania. World Archaeol 17:131-147.

VIVAR, G. 1966. Crónica y relación copiosa y verdadera de los reinos de Chile. Santiago: Fondo Histórico y Bibliográfico José Toribio Medina. Santiago: Instituto Geográfico Militar. 231 pp.

Original recibido el 2 de septiembre de 2011; aceptado el 21 de noviembre de 2011. 